\title{
Investigating the round air jet dynamics at low Reynolds numbers
}

\author{
Vadim Lemanov ${ }^{1}$, Konstantin Sharov ${ }^{1 *}$, Andrey Shumeiko ${ }^{1}$, and Nadezhda Gorinovich ${ }^{1,2}$ \\ ${ }^{1}$ Kutateladze Institute of Thermophysics SB RAS, 630090, 1 Ac. Lavrentiev Ave., Novosibirsk, \\ Russia \\ ${ }^{2}$ Novosibirsk State Technical University, 630073, 20 Karl Marks Ave., Novosibirsk, Russia
}

\begin{abstract}
The laminar-turbulent transition in a round jet flowing from a cylindrical channel with the diameter of $3.2 \mathrm{~mm}$ was studied experimentally. In experiments, the range of Reynolds numbers determined by the mean-flow velocity was $\operatorname{Re}=U d / v=700-12000$. The measurements were carried out using a PIV system and one-component hot-wire anemometer. The profiles of average velocities and their pulsations in the zone of laminar-turbulent transition were obtained, as well as axial distributions of longitudinal velocity and pulsations of longitudinal velocity.
\end{abstract}

\section{Introduction}

It is known that jets are unstable at low Reynolds numbers $\mathrm{Re}=10-30$ [1], however, the transition to turbulence occurs at significantly higher values of $\operatorname{Re}=30-2000[2,3]$. The gas jets flowing from the contoured nozzles and at large Reynolds numbers $\operatorname{Re}>10^{4}$ are studied. It is known that the initial conditions significantly affect the dynamics of the flow development in the jet. The laminar-turbulent transitions occurring in the jet flowing from the nozzle and cylindrical channel differ. This is due to the difference in the initial velocity profiles. There are almost no publications on the jets from the long channels, although in practice, such cases are rather frequent.

The feature of extended channels is that the laminar, turbulent and transient flows can occur there, and this fundamentally determines characteristics of the jets flowing from these channels. By now, the influence of these factors has been studied insufficiently.

\section{Experimental methods and facilities}

The submerged jet was formed by a round metal channel with diameter $d=3.2 \mathrm{~mm}$ and length of $100 \mathrm{~d}$. Air from the high-pressure manifold was sent to the channel through a reducer and valve of fine adjustment. The air flow rate was measured by rotameters Krohne DK 800.

\footnotetext{
*Corresponding author: sharov@,itp.nsc.ru
} 
The air jet flowed into the flooded air space (a plexiglass flow chamber of 150x150x400 $\mathrm{mm}$ ). To visualize the flow and measure the velocity fields, a water aerosol with particle diameter of 1-2 $\mu \mathrm{m}$ was added into the flow. The surrounding space in the flow chamber was also filled with a mixture of air and water aerosol. When using the hot-wire anemometer, the compressed air line, feeding the aerosol generator, was turned off. The automated flow controller EL-Flow of Bronkhorst was included into the compressed air line feeding the main flow instead of the DK 800 rotameter.

The experiments were carried out using a PIV system, including digital camera, solidstate pulsed laser, pulse synchronizer and computer. Resolution of matrix of the digital camera was $1024 \times 1360$ pixels, laser pulse duration was $5 \mathrm{~ns}$, radiation energy was $50 \mathrm{~mJ}$, laser sheet thickness was $0.2 \mathrm{~mm}$, and minimal time between frames was $20 \mu \mathrm{s}$. In experiments with PIV, the measuring region was $15 \times 20 \mathrm{~mm}$, and the design area corresponded to the size of $0.53 \times 0.53 \mathrm{~mm}$.

Measurements with a hot-wire anemometer were carried out by the DISA 55M system consisting of: single-wire sensor 55P11 with wire diameter of $8 \mu \mathrm{m}$, measuring bridge, and voltmeter of mean square values. The signal received from the hot-wire anemometer was converted by a linearizer.

\section{Results}

\subsection{Initial conditions}

The initial conditions are very important for the jets, they are distributions of average velocities and velocity pulsations at the outlet of the channel, forming a round jet.
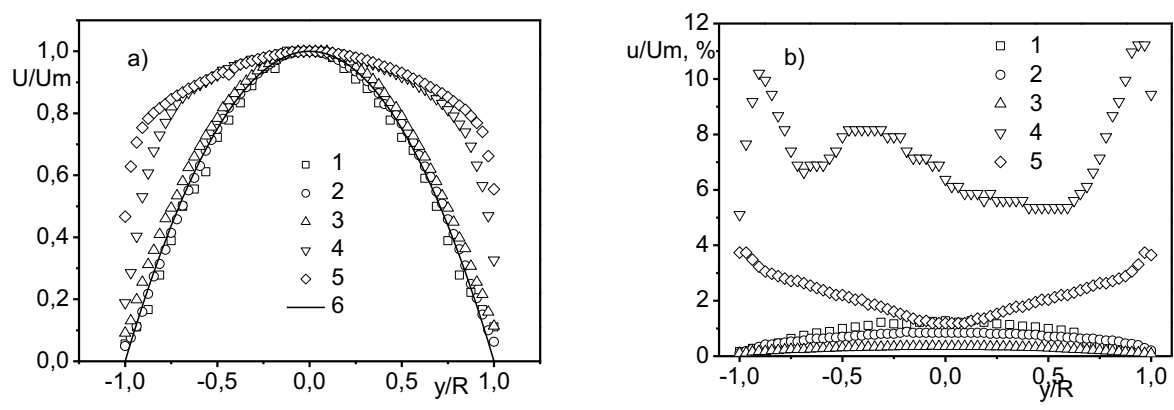

Fig. 1. Profiles of average velocities (a) and turbulent pulsations (b) at the edge of a cylindrical channel: $1-\operatorname{Re}=700 ; 2-1100 ; 3-3990 ; 4-4060 ; 5-12100$; line 6 - Poiseuille profile.

The measurements were carried out by a hot-wire anemometer at a distance of $0.5 \mathrm{~mm}$ from the tube edge.

Since the length of the channel, which formed a round submerged jet, is sufficiently large, then for $\mathrm{Re}<2000$ in the initial cross-section of the jet, velocity distribution measured along the cross-section must be close to the known Poiseuille profile (Fig. 1a, line 6) for the flow in cylindrical channels. Measurements on the channel edge (Fig. 1a) showed that the average velocity profile is well described by the well-known Poiseuille dependence, under the experimental conditions of up to $\operatorname{Re} \sim 4000$.

This indicates that the flow in the channel was laminar. The corresponding pulsation profiles are shown in Fig. 1b. It can be seen that for the laminar flow the degree of turbulence does not exceed $1.3 \%$. With a further increase in the Re number, the laminarturbulent transition starts forming in the channel. The velocity profile becomes smoother 
(Fig. 1a, symbols 4), and pulsations increase significantly (Fig. 1b, symbols 4) near the walls and channel axis. At $\operatorname{Re} \sim 12000$ the fully developed turbulent flow is observed on the channel edge.

\subsection{Laminar-turbulent transition in a round jet}

Distributions of longitudinal average velocities and longitudinal velocity pulsations on the jet axis measured by the hot-wire anemometer are shown in Fig. 2a and Fig. 2b, respectively. For $\mathrm{Re}<1000$, the average velocity on the jet axis decreases monotonically along the jet. At that, velocity pulsations here increase insignificantly at large distances from the tube edge. This indicates that the jet remains laminar along the entire length. Starting from $\operatorname{Re}=1000$, there is a noticeable increase in velocity pulsations (Fig. 2b, symbols 2 ) at a distance of $\sim 20 \mathrm{x} / \mathrm{d}$ from the cylindrical channel outlet. This indicates the beginning of transient processes in the jet.

With further increase in $\mathrm{Re}$, in spite of the fact that the flow in the channel remains laminar, the laminar-turbulent transition is observed in the jet. There is a distinct peak in distribution of axial pulsations (Fig. 2b, symbols 3), then, there is a rapid drop. Distribution of the average velocity on the jet axis also shows a sharp decrease (Fig. 2a, symbols 3). This behavior is caused by a significant increase in the jet half-apex angle after the zone of laminar-turbulent transition, which causes a decrease in the average velocity on the axis.
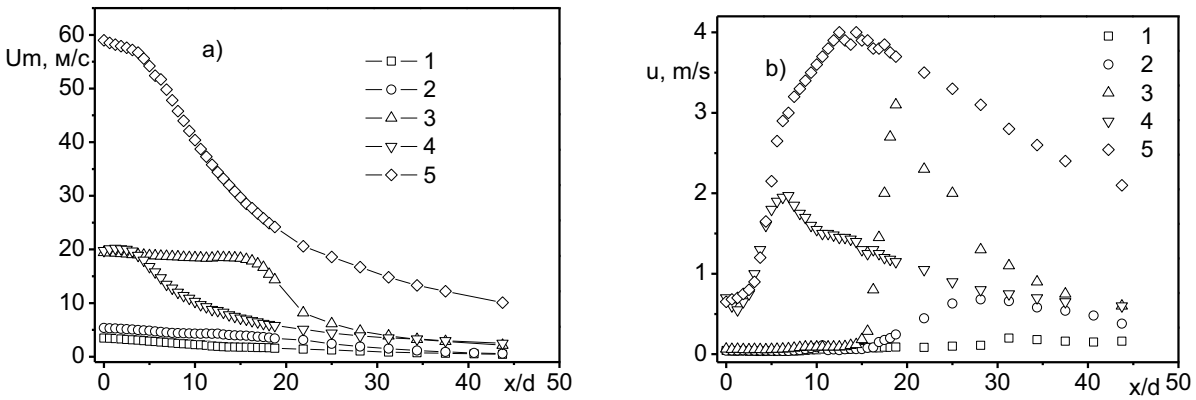

Fig. 2. Distributions of average longitudinal velocities (a) and turbulent longitudinal pulsations (b) on the jet axis along its length: $1-\operatorname{Re}=700 ; 2-1100 ; 3-3990 ; 4-4060 ; 5-12100$.

In the range of $\operatorname{Re} \sim 1000-4000$, the laminar-turbulent transition was observed in the jet, although the flow in the cylindrical channel was still laminar. The position of the zone of laminar-turbulent transition in the jet depended on the Re number, and with it's increase it was shifted to the edge of channel. When the Re numbers corresponding to the transient processes in the cylindrical channels were achieved, as it can be seen in Fig. 2a, symbols 4, the laminar region of the jet flow became minimal. Fig. $2 b$, symbols 4 shows attenuation of pulsations in the laminar flow and their sharp increase during transition.

The transition to turbulence in a cylindrical channel leads to an increase in pulsations from the very edge of the tube (Fig. 2b, symbols 5). At some distance, the pulsations reach a maximum, and their recession begins. The average velocity (Fig. 2a, symbols 5) also shows a drop from the very outlet from the cylindrical channel. In this case, this behavior is caused by the turbulent processes.

The profiles of average velocity and pulsations in the round jets were measured by the PIV system. At $\operatorname{Re}=1700$, which was determined from the mean-flow velocity, the laminar-turbulent transition in the jet started at a distance of about $20 \mathrm{x} / \mathrm{d}$. The transition 
point determined as for the hot-wire measurements and corresponding to the maximum of turbulent pulsations was located at the distance of $25 x / d$ from the cylindrical channel edge, where the jet flowed out. In experiments, the measurements were carried out at the site, which covered the distance from 20 to $40 \mathrm{x} / \mathrm{d}$. Distributions of the average and pulsation velocity components were obtained during the flow transition from the laminar to turbulent regime.

Distributions of average velocities tend to become smoother to the end of the laminarturbulent transition. This is explained by an increase in the angle of jet half-apex at transition to the turbulent flow. On the jet axis, the turbulence degree is not large and it makes up several percents. At transition, pulsations on the axis intensively increase, reach a maximum, then there is a small decrease and simultaneously the pulsations profile becomes smoother. Longitudinal and transverse pulsations behave similarly.

The data on Reynolds stresses have been also obtained. They save the zero value on the jet axis along the transition zone and have one extremum in each middle part of the jet mixture layers.

\section{Conclusions}

The air jet flowing out of a cylindrical channel with large length at low Reynolds numbers has been studied. The effect of initial conditions, such as distributions of average velocities and velocity pulsations at the outlet of the channel, on development of submerged jets has been investigated. Distributions of average axial velocities and pulsations along the jet were obtained in a wide range of Reynolds numbers $(\operatorname{Re}=700-12000)$. The profiles of average velocity, as well as profiles of longitudinal and transverse pulsations in the zone of laminarturbulent transition in the jet were obtained. It is shown that the laminar-turbulent transition in a jet is associated with the laminar-turbulent transition in a channel. Data on transverse velocity pulsations and Reynolds stresses in the zone of laminar-turbulent transition may be considered as novel.

The work was partially supported by the Russian Foundation for Basic Research (grant 17-08-00958).

\section{References}

1. G.N. Abramovich, The Theory of Turbulent Jets (M.I.T. Press, 1963)

2. V.V. Lemanov, V.I. Terekhov, K.A. Sharov, A.A. Shumeiko, Tech. Phys. Letters 39, $421(2013)$

3. V.M. Aniskin, V.V. Lemanov, N.A. Maslov, K.A. Mukhin, V.I. Terekhov, K.A. Sharov, Tech. Phys. Letters 41, 46 (2015) 